Nonlinear Processes in Geophysics (2002) 9: 11-23

Nonlinear Processes

in Geophysics

CE European Geophysical Society 2002

\title{
Impulse exchange at the surface of the ocean and the fractal dimension of drifter trajectories
}

\author{
D. M. Summers \\ School of Mathematics and Statistics, Napier University, 219 Colinton Road, Edinburgh EH14 1DJ, UK
}

Received: 17 April 2001 - Accepted: 15 May 2001

\begin{abstract}
An impulse-based model is developed to represent a coupling between turbulent flow in the atmosphere and turbulent flow in the ocean. In particular, it is argued that the atmosphere flowing horizontally over the ocean surface generates a velocity fluctuation field in the latter's near-surface flow. The mechanism for this can be understood kinematically in terms of an exchange of tangentially-oriented fluid impulse at the air-sea interface. We represent this exchange numerically through the creation of Lagrangian elements of impulse density. An indication of the efficacy of such a model would lie in its ability to predict the observed fractal dimension of lateral trajectories of submerged floats set adrift in the ocean. To this end, we examine the geometry of lateral tracer-paths determined from the present model.
\end{abstract}

\section{Introduction}

The atmosphere over an ocean (for heights up to $1000 \mathrm{~m}$ ) consists of a turbulent boundary layer; furthermore, the ocean itself (for depths down to $100 \mathrm{~m}$ ) is characterized by a field of relatively small-scale turbulence. Despite the obvious differences in character between these two turbulent processes, it would seem natural to ask whether they are coupled in some causal way.

In the following, we develop a Lagrangian model of the equilibrium of forces at the interface, $\partial \mathcal{D}$, between a flat, stationary ocean and the atmosphere. We express this as an exchange of impulse. We understand impulse $\boldsymbol{I}$ in a standard way as the time-integral of force, $\boldsymbol{F}$, in the limit of vanishing time-interval, $\delta t$, i.e. as the integral

$\boldsymbol{I}\left(\boldsymbol{r}, t_{o}\right)=\lim _{\delta t \rightarrow 0} \int_{t_{o}}^{t_{o}+\delta t} \boldsymbol{F}\left(\boldsymbol{r}, t^{\prime}\right) d t^{\prime}$,

where $\boldsymbol{F}\left(\boldsymbol{r}, t^{\prime}\right)$ represents the force field acting at the point $\boldsymbol{r} \in \partial \mathcal{D}$, at time, $t^{\prime}$. An formulation in terms of impulse is a

Correspondence to: D. M. Summers

(davids@maths.napier.ac.uk) useful way to represent the present physical problem of the interaction between a boundary and an impinging fluid flow. By considering fixed instants of time $(\delta t \rightarrow 0)$, we draw some insight from problems and solutions associated with static equilibria. The equilibrium of an interface between two relatively moving, materially homogeneous, fluid media can be understood in the following terms: flow in one medium imparts an impulse to an adjoining medium at the mutual interface between them. In turn, the adjoining medium imparts an equilibriating impulse to the first medium. For horizontal flow over a planar ocean, this may be stated specifically: wind flowing tangentially over the ocean imparts an impulse to the ocean; the ocean imparts an equilibriating impulse to the atmosphere. The effect of this exchange on the atmosphere is the formation of the atmospheric boundary layer; the effect on the ocean is a velocity fluctuation field evolving downwards into the ocean.

The foregoing exchange of impulse is conducted in a way that is consistent with the boundary conditions to be satisfied at $\partial \mathcal{D}$, where we require that the two fluids do not interpenetrate, and that there is no "slip" at their mutual boundary. This latter "no-slip" condition is consistent with the creation of an impulse in the form of a thin vortex doublet sheet, coincident with the interface (there is a close relationship between elements of impulse of compact support and vortex elements; see Summers, 2000a, b, 2001). Once an impulse is created, it proceeds to evolve from the interface into the surrounding fluid according to an equation of motion introduced by Oseledets (1988). Turbulent flow has been associated with this evolution; (see Buttke, 1993; Buttke and Chorin, 1993; Chorin, 1994; Smereka , 1996; Summers, 2001). The turbulence fluctuation field can be attributed to the macroscopic effect of an evolving ensemble of vortical structures in a fluid. In the present case of a created impulse at the surface of an ocean, the relevant evolution is that of a system of vortex doublet sheets.

One specific signature of the velocity fluctuation field in the ocean has been observed for horizontal scales of 10$200 \mathrm{~km}$, and for vertical depth scales of up to $100 \mathrm{~m}$. This 
arises from the fact that experimentally observed trajectories of passive tracers in the horizontal flow near the ocean surface describe fractal curves; these curves have a Hausdorff dimension of about 1.3. Such trajectories have been observed, for example, in the Kuroshio Extension in the Pacific Ocean (Osborne et al., 1989) and in the northern Atlantic Ocean (Sanderson and Booth, 1991). The global distribution of the observations suggests that some degree of universality is obtained. The observations have attracted interest from the perspective of dynamical systems theory (Osborne and Caponio, 1990). The fractal character of such trajectories can be numerically reproduced in particle models based on fractional-order Brownian motion in a plane (see, for example, Addison et al., 1997).

Tracer trajectories in the ocean do not exhibit the Hausdorff dimension expected of a purely harmonic process, such as that resulting from a linear superposition of waves (such a process would have a fractal dimension of 1). In addition the trajectories are not consistent with simple Fickian diffusion (with dimension 2). The observed non-integer dimension has been widely interpreted as a signature of ocean turbulence (for example, see Osborne and Caponio, 1990).

The surface of the ocean is a complex system. The prospect might seem remote that one could isolate (in a mathematical model) vorticity-induced velocity fluctuations from the remaining flow. However, we shall see (Sect. 2) that progress can be made if we exploit a decomposition of the velocity field which was introduced by Kuz'min (1983). When this decomposition is applied in the context of an ocean surface, it effectively separates the flow problem into that associated with a "vortex sheet" at the interface (whose selfinteraction and resulting instability are classically related to surface wave propagation; (for example, see article 239 of Lamb, 1932), and that associated with velocity fluctuations induced by a distribution of sub-macroscopic vortex doubletsheets decending into the ocean.

\section{Flow as an evolution of impulse}

A point source of impulse may serve as a Lagrangian object which induces a velocity field with dipolar structure (see Sects. 2.4, 2.5 and 7.2 of Batchelor, 1967), or (Sect. 11.2 of Lighthill, 1986). In two dimensions, this is a vortex pair or doublet. An ensemble of such objects constitutes, in the absence of viscosity, a Hamiltonian system (Roberts, 1972); a dissipative perturbation of such a system reflects the dynamics of flow with slight viscosity. In order to develop an ocean model based on such elements for wind flow over a stationary boundary, we need to determine the relationship between the impulse and the relevant boundary conditions.

This is achieved by considering the decomposition introduced by Kuz'min (1983):

$\boldsymbol{u}=\boldsymbol{m}+\nabla \phi$,

where $\boldsymbol{m}$ is a vector field (normalized with respect to mass density) which is of compact support and has non-vanishing divergence. Buttke (1993) (see also Buttke and Chorin, 1993) demonstrates that $\boldsymbol{m}$ is, by virtue of its compact support, related to the impulse through the volume integral

$\mathbf{I}(\mathbf{r})=\rho \int_{V} \boldsymbol{m} d V$.

This is to say that $\boldsymbol{m}$ is a volume density of impulse, $\mathbf{I} . \nabla \phi$ is an irrotational extensive field. We read Eq. (2) as a material decomposition of $\boldsymbol{u}$ into a local, source-like component ( $\boldsymbol{m})$ and into a non-local, ensemble-averaged field $(\nabla \phi)$. Equation (2) can be understood as a Hodge decomposition of $\boldsymbol{m}$ into divergence-free $(\boldsymbol{u})$ and curl-free $(\boldsymbol{\nabla} \phi)$ components. We note from Eq. (2) that vorticity defined through $\boldsymbol{\xi}=\boldsymbol{\nabla} \times \boldsymbol{m}$ also has compact support.

Oseledets (1988) determined an equation of motion for $\boldsymbol{m}$ (see also Buttke, 1993) by substituting Eq. (2) into the Navier-Stokes equation to obtain

$$
\frac{D \boldsymbol{m}}{D t}=-(\nabla \boldsymbol{u})^{T} \boldsymbol{m}+v \Delta \boldsymbol{m}
$$

(where we have invoked vector identities and have chosen the geometric gauge condition described by Russo and Smereke, 1999). The $i j$-element of the matrix $(\nabla \mathbf{u})^{T}$ is $\partial u_{j} / \partial x_{i}$; the parameter $v=\mu / \rho$ denotes the kinematic viscosity of the fluid.

Since our intention is to represent the impulsive action at the ocean-atmosphere interface at a fixed instant, we take the divergence of Eq. (2) to determine the Poisson equation

$\Delta \phi=-\nabla \cdot \boldsymbol{m}$.

We infer from this that if we have a known distribution of impulse density in an atmospheric flow and if we have gradient boundary conditions for $\phi$ at the ocean-atmosphere interface, then we can determine $\phi$ in the atmosphere to an additive constant; hence, we can determine $\nabla \phi$ there uniquely, and thereby specify the decomposition Eq. (2). In the following section, we develop a relevant gradient boundary condition for $\phi$ (see also Summers, 2000a, b, 2001).

\section{The ocean-atmosphere interface}

\subsection{Boundary conditions in $\mathbf{R}^{3}$}

We will assume at the interface between the air and ocean that the two fluids do not interpenetrate at molecular scale; furthermore, we assume the two fluids adhere at their common surface of contact, i.e. they experience a zero "slip" at that point. For our purpose of modelling the background ocean turbulence, we consider an idealized cartesian domain of flow $(x, y, z) \in \mathbb{R}^{3}$, where the interface is taken to be the plane $z=0$. For the purpose of showing the growth of an atmospheric boundary layer, and to give the problem a bounded expression, we consider a uniform-profile atmospheric flow (occupying $z>0$ ) of magnitude $U$ to begin impulsively at $t=0$, and to flow steadily in the positive $x$-direction over the surface of a geometrically flat ocean, 
occupying the region $z \leq 0$. We apply the viscous boundary conditions over a representative finite lateral domain in $x>0$ characterized by a lateral length scale of $L_{o}$. We denote the uniform density of the atmosphere as $\rho_{A}$, and that of the ocean as $\rho_{W}$, and consider flow in the interiors of both media to be incompressible. In this situation, consistent with the previous conditions at $z=0$, the impinging wind imparts an impulse to the ocean, an impulse which is oriented tangentially in the positive $x$-direction. The ocean will impart an equilibriating impulse to the atmosphere, an impulse which will be oriented in the negative $x$-direction.

We assume the ocean is otherwise stationary at macroscopic scale (i.e. we neglect drift currents). The problem as stated has two-dimensional symmetry; we confine our immediate attention to the $x-z$ plane (in Sect. 6 , we consider the $x-y$ plane).

\subsection{Two-dimensional statement in the $x-z$ plane}

The horizontal flow of the atmosphere over the stationary planar interface $\partial \mathcal{D}$, satisfies the boundary condition $\boldsymbol{u}=\mathbf{0}$, which we express as the union of impermeability and no-slip conditions, i.e. as

$\left.\begin{array}{l}\hat{z} \cdot \boldsymbol{u}=0 \\ \hat{\boldsymbol{x}} \cdot \boldsymbol{u}=0\end{array}\right\}$ on $\partial \mathcal{D}$

where $\hat{z}$ is a unit outward (relative to the ocean) normal to $\partial \mathcal{D}$ and where we understand the unit vector $\hat{\boldsymbol{x}}$ to be tangential to the ocean surface.

We substitute Eq. (2) into Eq. (6) to determine

$\left.\begin{array}{l}\boldsymbol{m}_{A} \cdot \hat{z}=-\partial \phi / \partial z \\ \boldsymbol{m}_{A} \cdot \hat{\boldsymbol{x}}=-\partial \phi / \partial x\end{array}\right\}$ on $\partial \mathcal{D}$,

where the subscript $A$ denotes the impulse on the atmosphere side of the interface.

Two complementary solutions to Eq. (5) result if we constrain $\boldsymbol{m}_{A}$ to be respectively tangential or normal to $\partial \mathcal{D}$. The former implies the condition $\partial \phi / \partial z=0$, known as Case I in Summers (2000a, b; 2001). The latter implies $\partial \phi / \partial x=0$ on $\partial \mathcal{D}$, known as Case II in Summers (2000a, b; 2001). In this way, we decompose the interaction of two fluids at their interface into two processes (the superposition of which expresses the complete interaction). If atmospheric flow is constrained to be in the $\hat{\boldsymbol{x}}$ direction and if the interface is constrained to occupy (over time) the surface $z=0$, then the impulse exchange is expressed by the former condition alone (i.e. by $\partial \phi / \partial z=0$ on $\partial \mathcal{D})$. Thus, we create a tangential impulse to effect no-slip at $z=0$.

\subsection{Impulse tangential to the interface}

We consider the impulse imparted to the atmosphere, $\boldsymbol{m}_{A}$, at $z=0^{+}$to be tangential to the ocean, i.e. we constrain the impulse in the atmosphere to satisfy $\boldsymbol{m}_{A} \cdot \hat{z}=0$ on $\partial \mathcal{D}$. Equations (7) now become

$$
\left.\begin{array}{l}
\boldsymbol{m}_{A} \cdot \hat{z}=0 \\
\boldsymbol{m}_{A} \cdot \hat{\boldsymbol{x}}=-\partial \phi / \partial x
\end{array}\right\} \text { on } \partial \mathcal{D}
$$

with the following gradient condition to be satisfied by $\phi$ on $\partial \mathcal{D}:$

$$
\frac{\partial \phi}{\partial z}=0 .
$$

(We also impose the condition that the solution be consistent with the incident flow field, i.e. we have $\boldsymbol{u}=U \hat{\boldsymbol{x}}$ at the lateral infinities of the half-space $z>0, x \rightarrow \pm \infty$.)

At $t=0$, given Eq. (9) we can solve Eq. (5) and hence, determine the gradient field $\nabla \phi$. We relate this to an irrotational field on $\partial \mathcal{D}$ through $\boldsymbol{u}^{*}=\partial \phi / \partial x \hat{\boldsymbol{x}}$, which satisfies the condition

$u^{*} \cdot \hat{z}=0$.

We hence deduce from Eq. (8) that the impulse density on $\partial \mathcal{D}$ satisfies $\boldsymbol{m}_{A}=-\boldsymbol{u}^{*}$ : this we understand to be the impulse imparted to the atmosphere by the ocean. The (Case I) impulse imparted to the atmosphere is consistent with establishing no-slip at $\partial \mathcal{D}$. The determination of $\boldsymbol{m}_{A}$ created at $\partial \mathcal{D}$, does not depend on the mass density $\rho_{A}$, but simply on the magnitude of $U$; furthermore, the subsequent evolution in $z>0$ only depends upon $\rho_{A}$ through the kinematic viscosity of the atmosphere, $v_{A}$.

\subsection{The response of the ocean}

While the evolution of $\boldsymbol{m}_{A}$ in $z>0$, according to Eq. (4), proceeds independently from the evolution of the impulse density in the ocean, $\boldsymbol{m}_{W}$, the two ensembles of impulse sheets are intimately related to each other at their point of creation on $\partial \mathcal{D}$. Specifically, $\boldsymbol{m}_{W}$ depends entirely on $\boldsymbol{m}_{A}$ (and on $\rho_{A}$ and $\rho_{W}$ ) for its existence. The evolving impulse in the ocean is causally derived from the motion of the atmosphere.

We deduce from Eq. (3) the equilibriating impulse $\mathbf{I}$ at $\partial \mathcal{D}$ by considering unit test volumes on either side of the interface; for equilibrium at a point on the interface, we require

$\rho_{A} \boldsymbol{m}_{A}+\rho_{W} \boldsymbol{m}_{W}=0$.

Hence, the impulse density $\boldsymbol{m}_{W}$, which must be imparted by the atmosphere to the ocean at their common interface, is

$\boldsymbol{m}_{W}=-\rho_{A} \boldsymbol{m}_{A} / \rho_{W}$

This tangential impulse density created in the ocean at $z=$ $0^{-}$will subsequently evolve downwards into the ocean. Thus, we present the problem of ocean-atmosphere coupling as a two-dimensional Lagrangian problem in a vertical section. We pursue this numerical model in Sect. 5.2.

\subsection{Simulating "dipole creation" in three dimensions}

In order to understand lateral flow in the ocean a model of a three-dimensional atmospheric boundary layer would be required. We note that, so far as the ocean itself is concerned, the atmosphere serves as an impulse generator at the surface $z=0$. Some understanding of the three-dimensional kinematics of an impulse in the ocean may be possible, if we 
represent the influence of the atmosphere through an "impulse creation boundary condition", i.e. at each time-step, we prescribe a distribution of wind-flow values on the ocean surface, and create an impulse doublet in response. Therefore, the wind is to be treated as a time-dependent creation boundary condition for an impulse in the ocean.

We can, for example, conceive of this surface condition in terms of a random surface distribution of wind flow directions (constrained to have a positive $x$-component, perhaps to capture a prevalent wind direction). This is intended to represent instantaneous wind flow over a surface expanse of ocean. Instead of evolving elements determined by partitioning a three-dimensional dipole sheet, we convert sheet elements into ameliorated point vortex dipoles with the same orientations of moment as the sheets from which they are derived, and with strengths, such that they induce the same velocity in the far field as these sheets. This resembles a standard hybrid approach used in vortex sheet methods to effect a matching of boundary layer Lagrangian sheet elements to point elements appropriate to an interior.

Such an expedient stops short of solving a Lagrangian three-dimensional boundary value problem. Even so, some insight into the qualitative character of the fluctuation field in the upper ocean may be anticipated from it. (The fractal dimension of drifter trajectories is one such qualitative characterization.) We pursue this numerically in Sect. 6.

\section{Thin-sheet Lagrangian elements of impulse $\left(\mathbb{R}^{2}\right)$}

We consider the viscous interaction of the wind and the ocean to be the only source of impulse density in the flow of either medium. This is an essential simplifying assumption of our model. We pose this as a two-dimensional problem in an $x-z$ vertical section. We represent the tangential impulse confined to the surface $\partial \mathcal{D}(z=0)$ as a thin doublet sheet. We can think of this as two vortex sheets, each with a linear vorticity density of the opposite sense and separated from each other by a distance of $d$. The doublet sheet derives from a thin-sheet limit

$$
\lim _{\substack{d \rightarrow 0 \\ \kappa \rightarrow \infty}} \kappa d=\mathcal{M}
$$

where the bounded limit $\mathcal{M}$ represents a tangential "impulse per unit length". Such a sheet can be partitioned and the resulting segment elements can be made to form a Lagrangian ensemble. Considering a segment of length $\ell$ aligned in the $x$-direction and centered at $\left(x_{o}, z_{o}\right)$, the velocity it induces at $(x, z)$ is determined in Eqs. (22) and (23) in Summers (2000a).

We ascribe to an impulse sheet at $z=0^{+}$an impulse per unit length of $\mathcal{M}_{A}=-u^{*} \ell$, where $u^{*}$ is the magnitude at $z=0$ of the irrotational field discussed in Sect. 3.2: this is evaluated at the mid-point of the sheet segment. The induced velocity fields are desingularized (see Krasny, 1986) by introducing an additive smoothing parameter into their respective denominators.
The choice of partition parameter $\ell$ may be based on a physical argument: choosing $\ell \sim O(v)$ is consistent with a flow in which translation due to inertial contributions is comparable to viscous displacement (see Summers, 2000a). This would represent the condition usually associated with the formation of a laminar boundary layer, for example. On the other hand, larger values of $\ell$ may be used to reflect the scale of surface roughness in the case of a "non-smooth" ocean (this could be chosen to reflect the scale of the surface wave pattern, for example).

The principle of impulse creation that we have described requires us to determine an irrotational field $\boldsymbol{u}^{*}=\nabla \phi$ in the atmosphere, satisfying $\boldsymbol{u}^{*} \cdot \hat{z}=0$ on $\partial \mathcal{D}$. A "nullfield" approach to solving this problem is described in Summers (2001). Note that in the present context, we do not need to determine an explicit solution to the Poisson Eq. (5); rather, we can invoke a method of images. The velocity field induced by an impulse dipole of moment $\boldsymbol{m}_{A}$, located at $\boldsymbol{r}=(x, y, z)$ and consistent with the condition $\boldsymbol{u}_{A} \cdot \hat{z}=0$ at $z=0$, is achieved by introducing an image dipole at $\boldsymbol{r}^{\prime}=(x, y,-z)$; the strength of this image will be $\boldsymbol{m}_{A}$, and its orientation will consitute a reflection across the interface $z=0$.

The argument also applies to the determination of the velocity in the ocean induced by an impulse dipole of moment $\boldsymbol{m}_{W}$, located at $\boldsymbol{r}=(x, y,-z)$, and consistent with the condition $\boldsymbol{u}_{W} \cdot \hat{z}=0$ at $z=0$. This is achieved by introducing an appropriate image dipole "in the atmosphere" at $\boldsymbol{r}^{\prime}=(x, y, z)$

\section{The transport of doublet sheets}

\subsection{Splitting strategy}

Once an impulse is created at a wall, it evolves into its respective flow interior according to the equation of motion Eq. (4). The dynamics of impulse are elucidated by the term $(\nabla \boldsymbol{u})^{T} \boldsymbol{m}$ in Eq. (4). This term can be expressed as the sum of symmetric and antisymmetric tensors, so that we write

$$
\begin{aligned}
\frac{D \boldsymbol{m}}{D t} & =-\underbrace{\frac{1}{2}\left((\boldsymbol{\nabla u})^{T}+(\nabla \boldsymbol{u})\right)}_{\text {extension }} \boldsymbol{m} \\
& -\underbrace{\frac{1}{2}\left((\boldsymbol{\nabla u})^{T}-(\nabla \boldsymbol{u})\right)}_{\text {rotation }} \boldsymbol{m}+v \Delta \boldsymbol{m} .
\end{aligned}
$$

This is to imply an evolution in the orientation and magnitude of impulse, as well as its advection in the stream, and its transport by viscous diffusion.

We pursue a Lagrangian numerical model based on a timesplitting of the equation of motion. We consider Eq. (4) as the simultaneous equation representing Euler advection,

$$
\frac{D \boldsymbol{m}}{D t}=0,
$$


and viscous diffusion,

$$
\frac{\partial \boldsymbol{m}}{\partial t}=v \Delta \boldsymbol{m} \text {. }
$$

Added to these is the evolution of impulse density strength, implied by

$$
\frac{\partial \boldsymbol{m}}{\partial t}=-(\nabla \boldsymbol{u})^{T} \boldsymbol{m} .
$$

This latter splitting bears analogy to a common treatment of vorticity stretching in three dimensions (discussed, for example, in Gustafson and Sethian, 1991).

The Euler Equation (13) is modelled numerically by evaluating the interaction between each pair of impulse elements (including their images). Note that these require modification in order to express the relative rotation between sheet elements (Summers, 2000a). We determine the advection of the resulting $n$-body system of impulse elements by using Runge-Kutta integration.

If we consider the evolution over a time-step interval $d t$, then Eq. (14) is modelled by considering a dissipative perturbation to the trajectories determined from Eq. (13), specifically by imparting to each impulse element a random displacement with zero mean and variance $2 v d t$. This represents a stochastic model of Browian motion (a random walk model). There are deterministic methods of modelling such diffusion, and these are numerically more accurate (see, for example, Fishelov, 1990; Bernard, 1995).

As elements of a partitioned doublet sheet evolve from the surface, they each actively induce a local contribution to the velocity field. Since part of this evolution is a viscous diffusion, the time variations of the ensemble-averaged induced velocity field will reflect a stochastic character. This field will, therefore, not be a manifestation of deterministic chaos; the non-integer fractal dimensions that we will determine (see Sect. 6) resemble, in this respect, those of the stochastic processes described by Osborne and Provenzale (1989).

\subsection{Numerical examples in the $x-z$ plane}

By way of illustrating the exchange in a familiar context, we consider the following reduced scale numerical example. The parameters will be chosen to conform to the neighbourhood of the Blasius profile: we conceive of a lateral domain on the order of $1 \mathrm{~m}$. We consider $\rho_{A} / \rho_{W}=0.001$, with both fluids being relatively viscous, i.e. we choose $v_{A}=0.01 \mathrm{~m}^{2} / \mathrm{s}$ and $v_{W}=0.01 \mathrm{~m}^{2} / \mathrm{s}$. We also choose parameters $\ell_{A}=0.001 \mathrm{~m}, \ell_{W}=0.001 \mathrm{~m}$ with $d t=0.05 \mathrm{~s}$. The smoothing parameter is in both cases 0.6 times the sheetsegment length. Our interest in examining this example relates to the fact that although the flow in the "air" medium is turbulent, it still preserves (in time average) something of the character of Blasius self-similarity. As such, the example serves to demonstrate the exchange of impulse in a numerically transparent setting.

The upper diagram in Fig. 1 represents the distribution of doublet sheets in this scale-model of the atmosphere and the ocean. In the atmosphere $(z>0)$, a turbulent boundary layer has developed over eight steps, which is represented by some 50000 elements; in the ocean, there is an evolving distribution of elements (9000 elements). Note that by virtue of Eq. (11), each element in $z<0$ carries a dipole moment 0.001 times its created counterpart in $z>0$. Furthermore, the implication of the lower particle density in $z<0$ (i.e. lower compared to the atmosphere) is that there is a smaller impulse per unit volume at that point. As one descends into the ocean, this density becomes less, indicating the effect of dissipation on the ensemble. The lower contour plot in Fig. 1 represents the velocity magnitude level curves in the respective media. In the atmosphere, the contours represent magnitude levels in the range of $0-1 \mathrm{~m} / \mathrm{s}$; in the ocean, the curves represent a range of $0-0.003 \mathrm{~m} / \mathrm{s}$.

\section{Trajectories of submerged drifters in near-surface flow}

\section{1 "Dipole creation" in three dimensions}

We consider that over a large expanse of ocean (thousands of kilometers) and over a long duration of time (weeks), the wind flow at any point on the ocean surface may change its speed and direction. As a consequence, the impulse imparted to the ocean at such a point may assume a variety of strengths and directions in the lateral plane. We model this situation by assuming a distribution of wind speeds which is random in magnitude and direction (although the wind can be constrained to veer within an angular pencil to reflect a prevalent wind direction). Given the consequential downwardly evolving and continuously created distribution of dipoles, we ask the question: what is the character of a passive tracer constrained to move in a lateral $x-y$ plane at depth $z_{D}$, in the midst of this impulse evolution?

To model passive tracers in an $x-y$ plane (i.e. moving in the projection - onto a plan section with the depth - of a three-dimensional velocity field), we propose to model the evolution in a simplified manner. Although stretching and rotation are explicitly modelled in the numerical experiment described in Sect. 5.2, these processes make a relatively small contribution to the evolution in the ocean. This situation is a consequence of Eq. (11): the strength of the doublet moments in $z<0$ is three orders of magnitude smaller than their counterparts in $z>0$ (having chosen $\rho_{A} / \rho_{W}=0.001$.)

In the context of the present three-dimensional case, we will model only the diffusion and advection of elements, i.e. we suppress the possibility for stretching and rotation in the ocean. Such an approximation may serve our limited purpose in modelling the trajectories of tracers. This expedient will greatly reduce the computational effort, although it does suppress the tendency towards large-scale instability, or the tendency towards coherence of elements to form macroscopic structures. These appear to be small effects, and in any case, they are of less importance to us as we pursue the creation of a sub-macroscopic fluctuation field. 

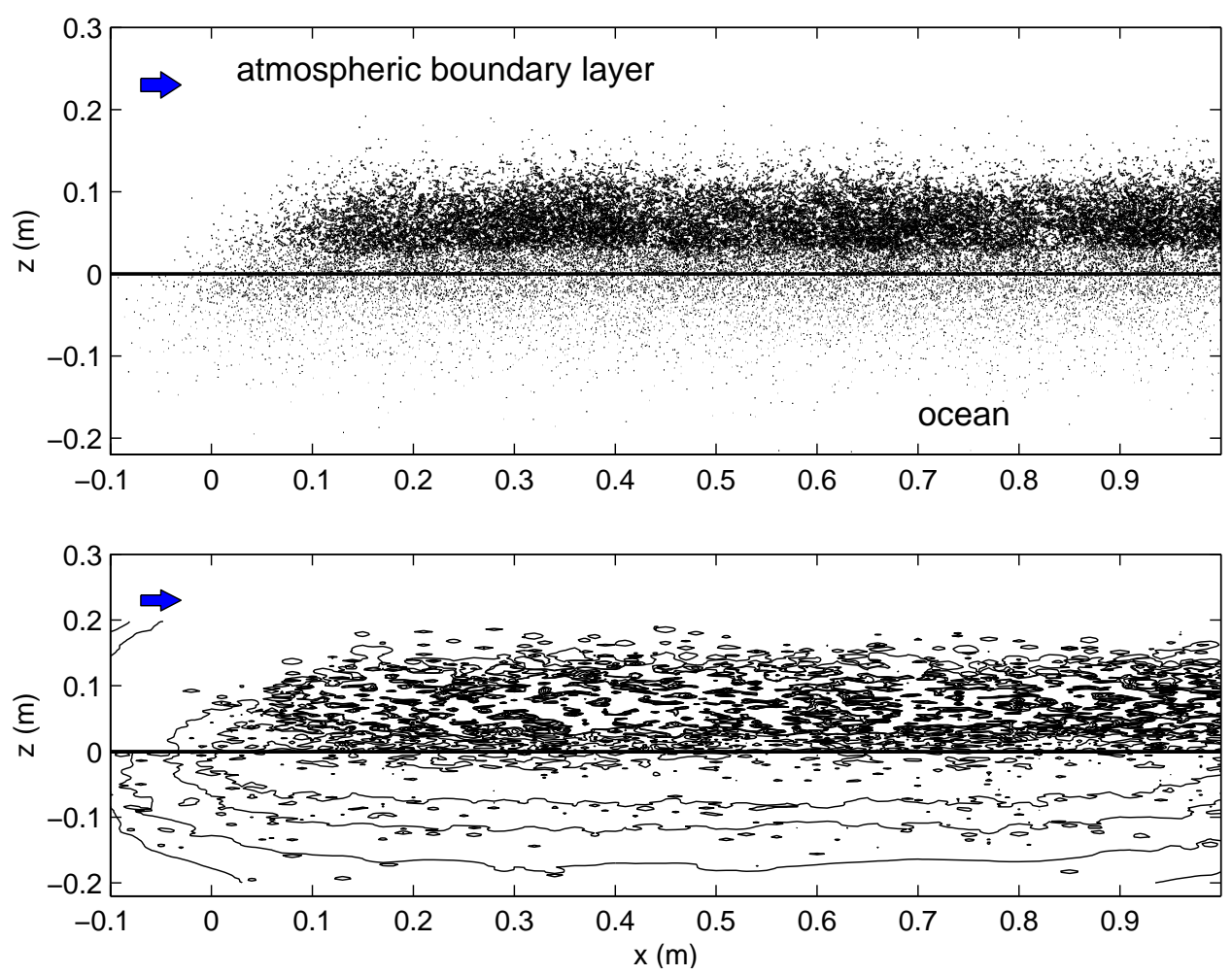

Fig. 1. (Above) Distribution of impulse elements in $y-z$ plane (i.e. in vertical section) after 8 time-steps have elapsed; (below) corresponding level curves of magnitude of the velocity field.

We shall create and partition an impulse doublet sheet, as described in Sect. 4. We will convert each element into an equivalent three-dimensional impulse dipole. The strength of this dipole $(\boldsymbol{M})$ will be chosen (numerically) so that the velocity field it induces in $\mathbf{R}^{3} \backslash \operatorname{supp}\{\boldsymbol{M}\}$ approximates that induced by its thin sheet counterpart. Figure 2 illustrates the comparison of field components (in the vertical $x-z$ plane) induced by a doublet sheet (of density $\mathcal{M}$ ) and its equivalent ameliorated point dipole element (of moment $\boldsymbol{M}$ ).

We release a passive marker into a procession of point impulse elements which evolves downwardly from the surface, at position $\left(x, y,-z_{D}\right)$, with the depth of the trajectory plane, $z_{D}$, chosen to approximate the ratio of drifter depth to the spatial extent of its ultimate trajectory. We allow the marker to negotiate its way in this plane through the (projected) velocity field induced by the evolving distribution of impulse dipoles. Specifically, the velocity field induced at $\boldsymbol{r}=\left(x, y,-z_{D}\right)$ by a vortex point dipole of moment $\boldsymbol{M}$, located at $\boldsymbol{r}_{o}=\left(x_{o}, y_{o},-z_{o}\right)$, is given by

$\boldsymbol{u}_{M}(\boldsymbol{r})=\frac{3(\boldsymbol{M} \cdot \hat{\boldsymbol{r}}) \hat{\boldsymbol{r}}-\boldsymbol{M}}{\left|\boldsymbol{r}-\boldsymbol{r}_{o}\right|^{3}}+\frac{3\left(\boldsymbol{M}^{\prime} \cdot \hat{\boldsymbol{r}}^{\prime}\right) \hat{\boldsymbol{r}}^{\prime}-\boldsymbol{M}^{\prime}}{\left|\boldsymbol{r}-\boldsymbol{r}_{o}^{\prime}\right|^{3}}$,

where $\hat{\boldsymbol{r}}$ is the unit vector in the direction $\boldsymbol{r}-\boldsymbol{r}_{o} ; \hat{\boldsymbol{r}}^{\prime}$ is the unit vector in the direction $\boldsymbol{r}-\boldsymbol{r}_{o}^{\prime}$, where $\boldsymbol{r}_{o}^{\prime}$ is the image point $\left(x_{o}, y_{o},+z_{o}\right)$. The vector $\boldsymbol{M}^{\prime}$ represents the reflection of $\boldsymbol{M}$ across $z=0$. In this experiment, $\boldsymbol{M}$ is understood to be horizontally oriented throughout the evolution. The simple Krasny-type desingularization of a sheet element mentioned in Sect. 4 is not possible for an amelioration of a point element (i.e. an element that will have no intrinsic spatial scale). Rather, the field of a point dipole (16) is to be desingularized through convolution with a Beale and Majda (1985) fourthorder smoothing kernel

$f(r / \delta)=1+\left(\frac{3}{2}\left(\frac{r}{\delta}\right)^{3}-1\right) e^{-(r / \delta)^{3}}$.

To match the field induced by a smoothed point dipole to that of a doublet sheet, the smoothing parameter, $\delta$, is adopted in terms of the partition parameter associated with the doublet sheet; typically, this smoothing parameter is chosen to be some fraction of $\ell$.

Although the velocity field induced by an ensemble of such dipoles is heterogeneous (especially at a scale comparable to $\delta$ ), it is nevertheless by design a desingularized and differentiable field. A passive tracer is impelled by this resultant velocity field alone. Although Lagrangian impulse elements undergo a Brownian motion that is consistent with the equation of motion for impulse (i.e. consistent with viscous diffusion), passive tracers are simply advected in the velocity induced by these elements; this emphasizes that no stochastic displacement is imparted to the passive tracers themselves.

\subsection{The fractal dimension of an irregular path}

As an initial experiment, we adopt directly the numerical parameterization associated with the model described in 


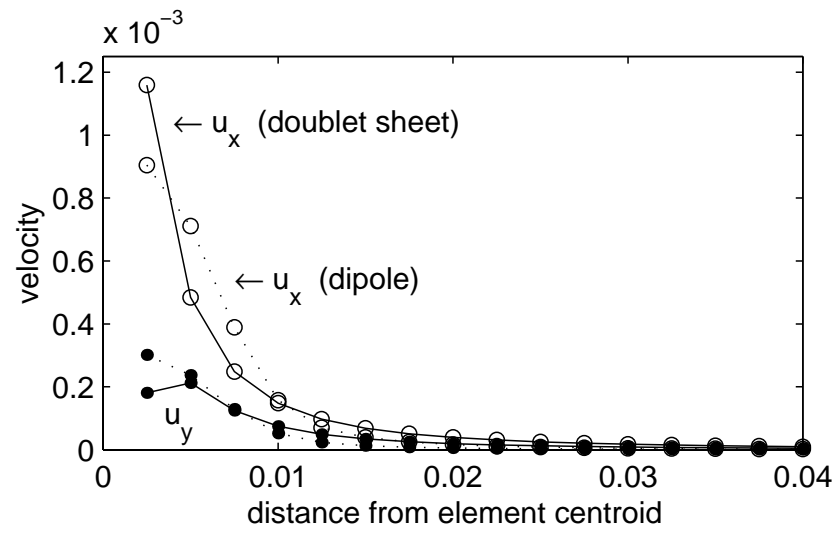

Fig. 2. Velocity components induced by a two-dimensional doublet sheet (solid line) and an equivalent three-dimensional impulse dipole (dotted line).

Sect. 6, namely $v_{W}=0.02, \ell_{W}=0.01 \mathrm{~m}$ with time-step interval $d t=0.05 \mathrm{~s}$. We conceive of the impulse generated at successive points of time at the surface $z=0$ as an ensemble of point impulse dipoles of uniform moment; these are randomly oriented at their points of creation in directions tangential to the ocean surface. The elements are positioned randomly (and uniformly) in a unit disc lying in $z=0$. The density of the ensemble created at each time-step will be chosen to be consistent with the density illustrated in Fig. 1. Thus, we create some 3000 elements at each timestep. In order to bound the number of Lagrangian elements, that accumulate in the time-evolution, we delete these after they have reached a depth of $35 z_{D}$. (A schematic representation of the model geometry is illustrated in Fig. 3).

The impulse moments are randomly oriented in the following way: the moment of each element is prescribed as $\left(M \cos \theta_{i}, M \sin \theta_{i}, 0\right)$ at $z=0$, where $\theta_{i}=-\frac{\pi}{2}+\pi q_{i}$, and $\left\{q_{i}\right\}$ is a set of random numbers with uniform distribution in $(0,1)$. Thus, the lateral orientation lies in a range $\theta_{i} \in\left[-\frac{\pi}{2},+\frac{\pi}{2}\right]$, i.e. the orientations are scattered in such a way that their moments are always positively oriented. This implies an element of anisotropy in the ensemble of dipole elements (see Sect. 6.4).

Figures $4 \mathrm{a}, \mathrm{b}$ and $\mathrm{c}$ illustrate respectively the trajectory of a typical passive marker after 30,100, and 1000 steps have elapsed, released from a point $\boldsymbol{r}=\left(0.4377 \times 10^{-4}, 0.3452 \times\right.$ $10^{-5},-0.2 \times 10^{-2}$ ). (The trajectories of Fig. 4 would represent, in an ocean context, the excursions from a drift current, i.e. they are the paths after a mean drift has been subtracted.)

We can seek to understand any implicit self-similarity in these trajectories by characterizing the paths as fractal curves of some Hausdorff dimension $D$. To approximate $D$, we can use a strategy introduced by Richardson (see Sect. 7 of Richardson, 1961) or (Sects. 5 and 28 of Mandelbrot, 1977): we "walk" a pair of dividers of fixed separation, $\eta$, along the trajectory in order to measure its length in a contiguous sequence of uniform segments, each of which has end-points

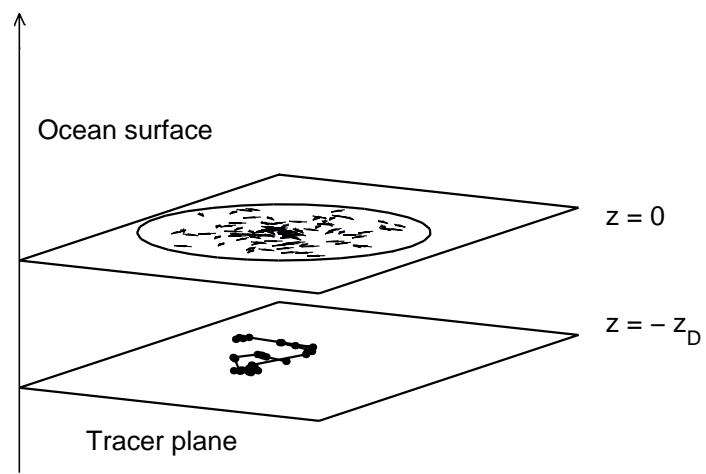

a

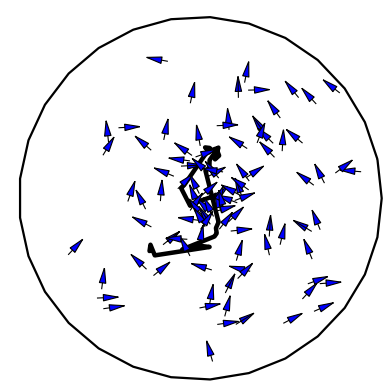

b

Plan view

Fig. 3. Schematic representation of dipole creation: (a) dipoles created at the ocean surface evolve into depth; a tracer is confined to move in the plane $z=-z_{D}$; (b) plan view (the prevalent wind direction points to "top of page").

that are coincident with the trajectory. Figure 5 illustrates the path of Fig. $4 b$ after this has been "measured" in this way, with $\eta=2 \times 10^{-5}$. An efficient algorithm (which we employ here) for constructing the sequence of line-segments that constitute this "measuring", is described by Rapaport (1985). The "length" of $\eta$-sequence, denoted by $L(\eta)$, is simply the product $N \eta$, where $N$ is the number of segments in the sequence.

A fractal dimension $D_{L}$, which approximates $D$, can be deduced from the relationship

$L(\eta)=A \eta^{1-D_{L}}$

(see Rapaport, 1985; Mandelbrot, 1977; Voss, 1988), where $A$ is a constant independent of $\eta$. In practical terms, one constructs a number of $\eta$-sequences corresponding to a range of divider separations; in this way, the function $L(\eta)$ is developed. If the trajectory exhibits strict self-similarity, then the exponent in Eq. (18) will be a constant from which $D_{L}$ can be deduced. In the context of numerical or physical experiments, one may expect such self-similarity to obtain in the average, specifically over an ensemble of realizations. In such a case, the self-similarity (or more accurately expressed, 


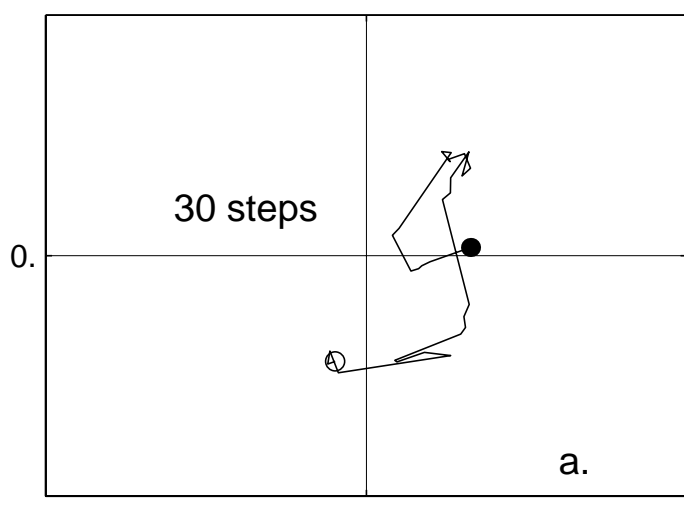

0.

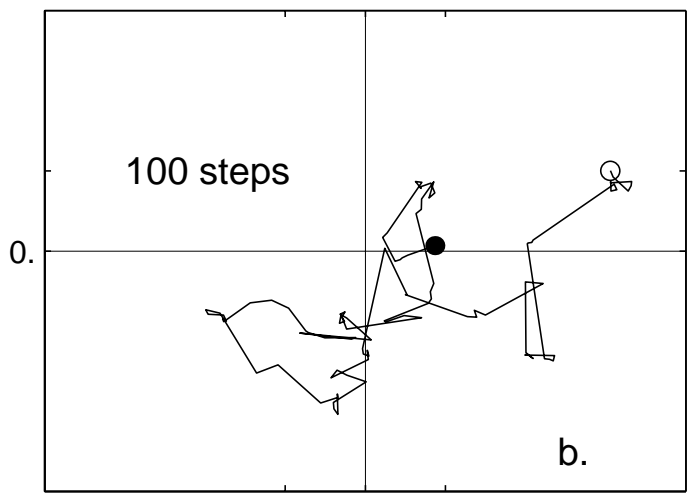

0.

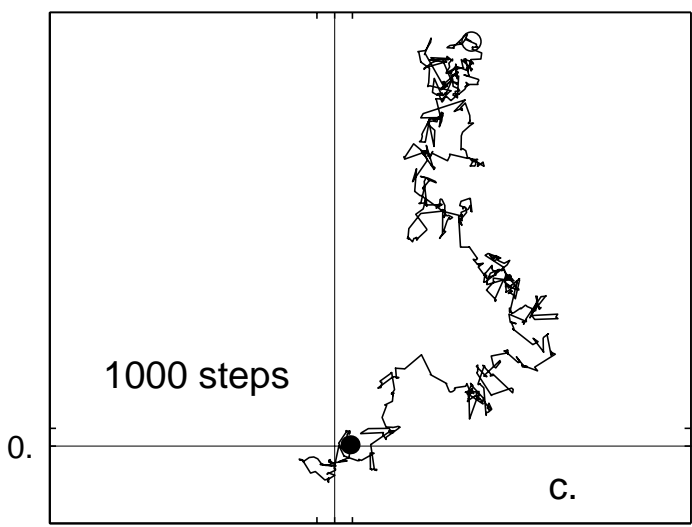

0.

Fig. 4. Passive tracer trajectories in the horizontal plane $z=$ -0.002 after 30, 100, and 1000 steps. Impulse dipoles are randomly distributed, and randomly oriented in a positive sense (i.e. such that $\theta \leq \pi$ ). Time interval is $d t=0.05 \mathrm{~s}$.

the "self-affinity") expresses itself through a linear regression applied to the log-log plot of $L(\eta)$ as a function of $\eta$.

The initial "linear part" of the positive gradient in the curves of Fig. 6 corresponds to divider separations that are too small to encompass the entire length of the trajectory in (arbitrarily) 500 contiguous segments. The following part of the curve, roughly with a zero gradient, corresponds to divider separations that do encompass the full length of the

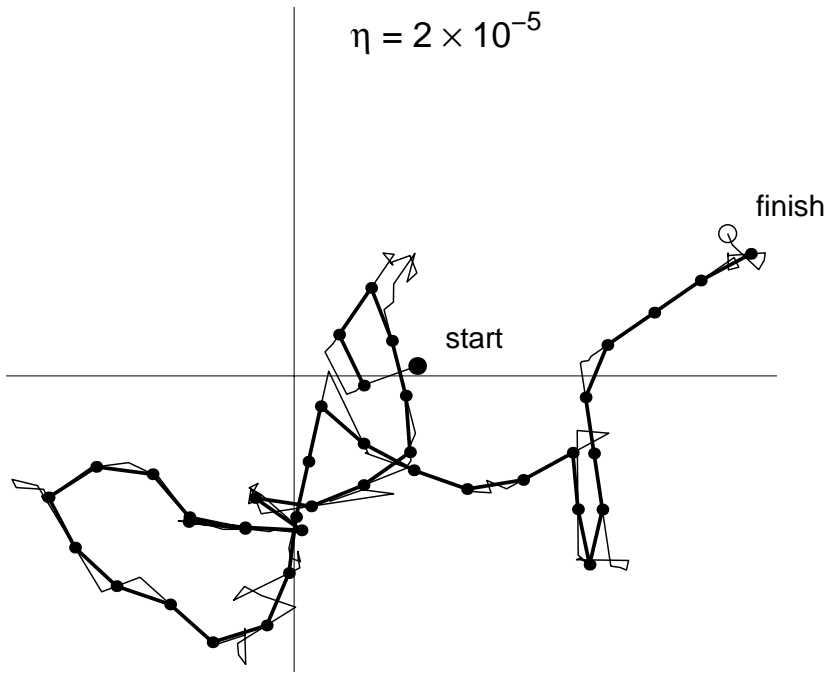

Fig. 5. The trajectory of Fig. 4b, "measured" using Rapaport's (1985) algorithm with the divider separation $\eta=2 \times 10^{-5}$.

trajectory, but are smaller in length than the smallest segment in the trajectory. It is the final part of the curve that yields through its gradient the parameter $D_{L}$.

In the case of the trajectories illustrated in Fig. 4, we determine $L(\eta)$ for a range of values of $\eta$. The fractal dimension is calculated from the log-log regression illustrated in Fig. 6. This exercise shows how the truncation of the data record for this particular trajectory affects the calculation of the fractal dimension: this varies between 1.2 and 1.5, depending upon the length of the measurement record (which ranges from 30 to 1000 locations, equispaced in time).

The data associated with the trajectory illustrated in Fig. 4 can be reconfigured in another obvious way. We could choose to sample the tracer locations at increasingly large time intervals. In other words, we could reconstruct the trajectory by taking every second node, every fifth node, etc. The fractal dimensions associated with these reconstructed paths are calculated (e.g. see Fig. 7) for sampling intervals of $d t=0.05,0.1,0.25,0.5,0.75$, and 1.0. The calculated fractal dimension $D_{L}$ varies between 1.2 and 1.5. The errors indicated are based on the regression residuals. This calculation is for a single trajectory; however, upon ensemble average, the error attached to the fractal dimension may well be larger. The results of the exercises associated with Figs. 6 and 7 are summarized in Fig. 8. The measurement sensitivity that is illustrated by these purely numerical exercises has been well understood for some time in experimental contexts.

We note the "curvature" of the $L(\eta)$ plots of Figs. 6 and 7. In the context of modelling molecular diffusion, Rapaport (1985) associates such curvature with a non-Gaussian distribution of vector displacements of active Lagrangian elements. In the present case, our distribution of created doublet elements has a directional bias, so we would expect this to be non-Gaussian.

We note that the calculated fractal dimensions indicated in these figures, determined from a very simple model, can 

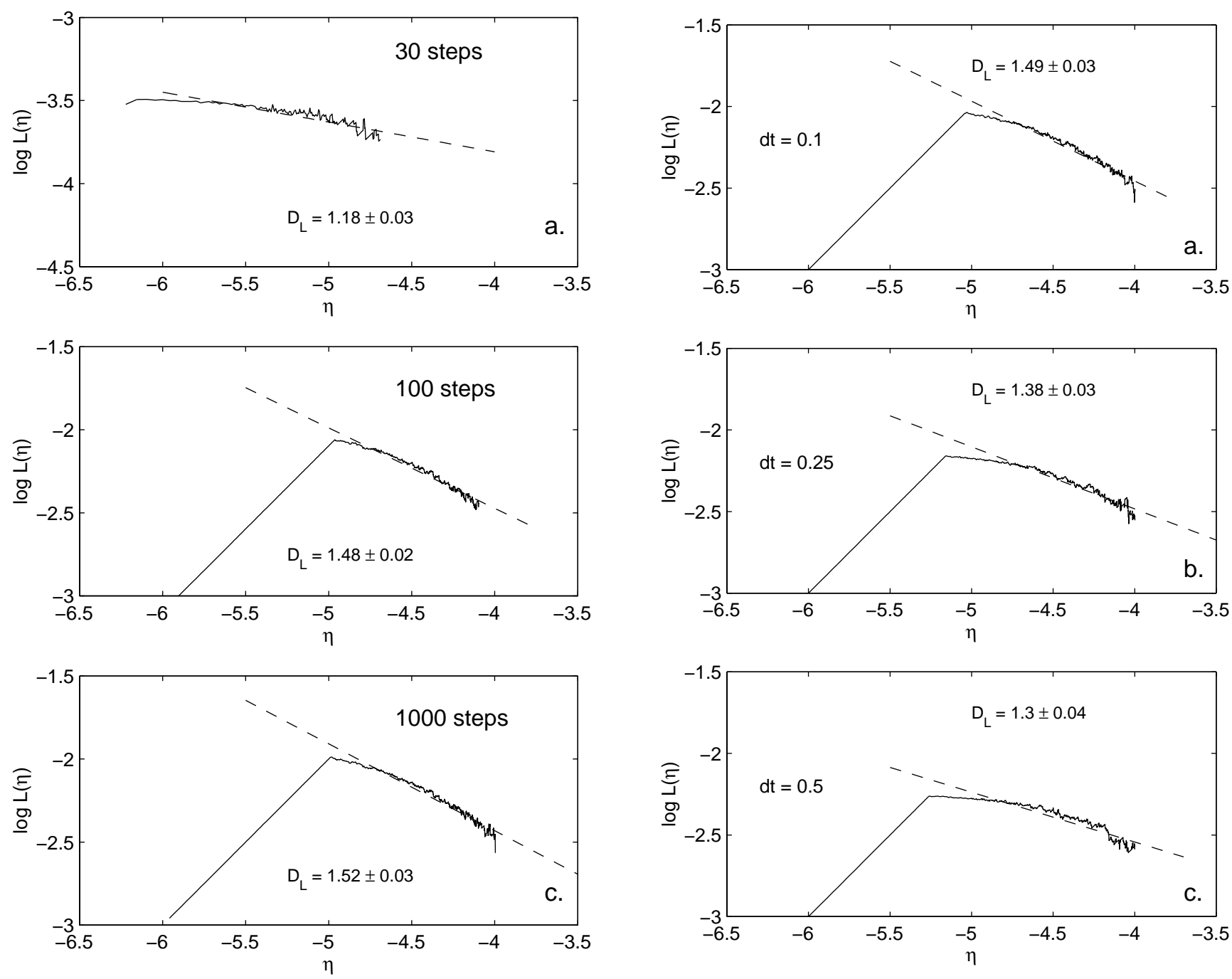

Fig. 6. For cases illustrated in Fig. 4: $\log -\log$ plot of length as a function of divider separation; regression indicates approximate fractal dimension in each case determined from Rapaport's algorithm.

be compared to that observed experimentally (at very different scales) from drifter trajectories in the ocean: for example, Osborne et al. (1989) obtained $D_{L}=1.27 \pm 0.06$ and Sanderson and Booth (1991) obtained $D_{L}=1.28 \pm 0.08$. We note, however, that Osborne et al. (1989) determined their data to be weakly multifractal in character, whereas we have pursued a monofractal treatment.

\subsection{Scaling up the problem to ocean dimensions}

The scales associated with the numerical experiments in Sect. 6.2 can be enlarged to reflect those of ocean drifter experiments. We consider a sheet length parameter of $\ell=$ $1000 \mathrm{~m}$, and an ensemble of impulse dipoles created in a disc of radius $10^{5} \mathrm{~m}(100 \mathrm{~km})$ randomly distributed in location and orientation, as described previously. We consider $v=10^{-6} \mathrm{~m} / \mathrm{s}^{2}$. The time-step is taken to be $d t=4320 \mathrm{~s}$, which is to say 1.2 hours. A passive tracer is released at

Fig. 7. The case of Fig. $4 \mathrm{c}$ re-sampled at coarsened time intervals, $d t=0.1,0.25$, and $0.5 \mathrm{~s}$.

a depth of $100 \mathrm{~m}$ from the surface and its trajectory at this depth is tracked over 70 steps (3.5 days). Figure 9a illustrates the resulting trajectory, where we infer (using Rapaport's algorithm) a fractal dimension of $D_{L}=1.19 \pm 0.08$.

Figure $9 \mathrm{~b}$ illustrates the trajectory for the same problem as Fig. 9a, except the magnitude of incident wind is increased from $1 \mathrm{~m} / \mathrm{s}$ to $5 \mathrm{~m} / \mathrm{s}$. After 50 steps, we infer a fractal dimension of $D_{L}=1.27 \pm 0.08$. We attach no significance to the closeness of this result to that derived from experimental measurement: rather, it is a matter of coincidence. Calculations based on alternative trajectories (released from other starting positions, for example) would result in another estimate of $D_{L}$. One expects in any given ocean trajectory the direction and strength of the incident wind-flow to vary over the duration of the experiment; furthermore, the "roughness scale" associated with the created impulse would also vary in time. From the present numerical exercise, we can develop, at best, a general expectation of the fractal dimension that is consistent with the proposed model. However, numerical 

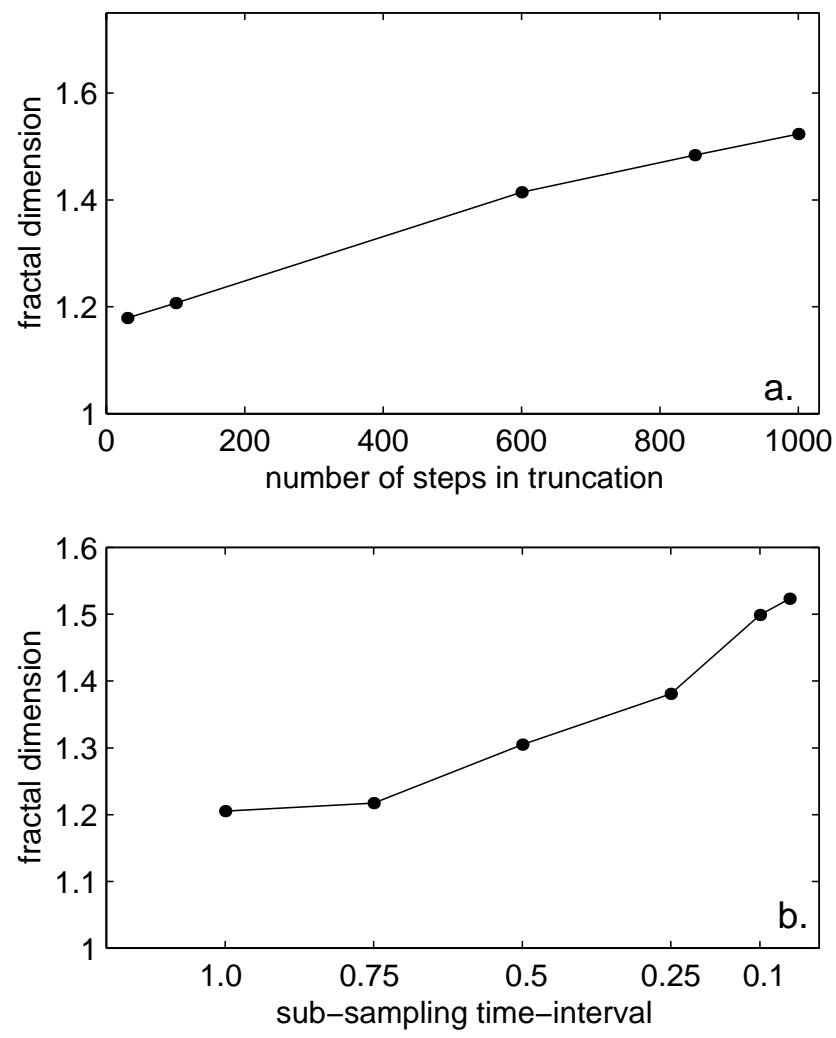

Fig. 8. Summary of effect on fractal dimension $D_{L}$ of the truncation and re-sampling of the trajectory of Fig. $4 \mathrm{c}$.

experiment suggests that the approximate fractal dimension that results from a variety of drifter starting positions falls well within the interval $D_{L} \in(1.0,2.0)$.

\subsection{Anisotropy of "wind-direction"}

One can conceive of a physical situation in which an impulse imparted to the ocean is generated by a prevailing wind flowing in one direction. The resulting distribution of impulse elements should mirror this as an anisotropy with respect to dipole moment orientation. This anisotropy can be accommodated in a graduated way into the present simple model. For example, if we choose $\theta_{i}=-\pi+2 \pi q_{i}$, then we have $\theta_{i} \in[-\pi,+\pi]$, which is to say that the orientations have an isotropic distribution. Generally, if for some $n \in[0,2)$, we choose $\theta_{i}=-\frac{\pi}{n}+\frac{2 \pi}{n} q_{i}$, then the moment vectors are aligned in a pencil defined by $\theta_{i} \in\left[-\frac{\pi}{n},+\frac{\pi}{n}\right]$.

Figure 10 shows the relationship between calculated fractal dimension and the pencil width (range in $\theta$ ) that controls the level of anisotropy in the model; this calculation is determined for 30-step trajectories with the numerical parameterization described in Sect. 6.2. The spread would seem to contract as the condition of isotropy is approached.
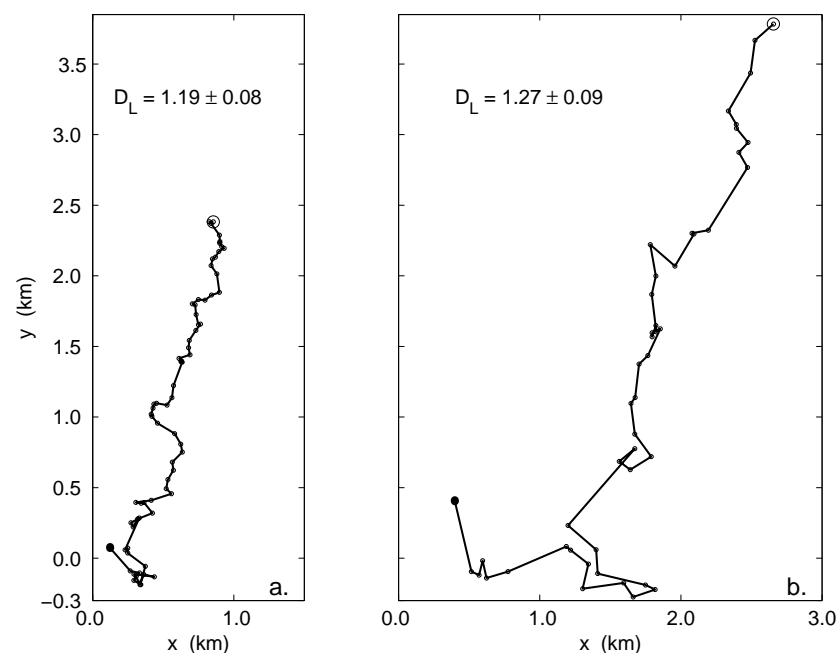

Fig. 9. (a) Trajectory over 70 steps $(d t=1.2$ days) at ocean scale, with $U=1 \mathrm{~m} / \mathrm{s}$; (b) trajectory over 50 steps for $U=5 \mathrm{~m} / \mathrm{s}$.

\section{Fractional Brownian motion (fBm)}

An ordinary Brownian motion random process $B(t)$ has the property that the sequence of increments $B\left(t_{o}+t\right)-B\left(t_{o}\right)$, with $t_{o}$ representing some multiple of $t$, i.e. $t_{o}=n t$, is a sequence of independent Gaussian random variables with zero mean and variance $t$. We write

$$
\left\langle\left[B\left(t_{o}+t\right)-B\left(t_{o}\right)\right]^{2}\right\rangle=A t,
$$

where $A$ is a constant. This is the principle which underlies the stochastic model of diffusion discussed in Sect. 5.1 .

The previous process can be generalized (Mandelbrot and Van Ness, 1968; Mandelbrot, 1999); we consider a process $B_{H}$ in which the variance satisfies

$$
\left\langle\left[B_{H}\left(t_{o}+t\right)-B_{H}\left(t_{o}\right)\right]^{2}\right\rangle=t^{2 H} \mathcal{V}_{H}
$$

with the coefficient $\mathcal{V}_{H}$ defined as

$$
\begin{aligned}
\mathcal{V}_{H} & =\frac{A}{\left[\Gamma\left(H+\frac{1}{2}\right)\right]^{2}}\left\{\int_{-\infty}^{0}\left[\left(1-t^{\prime}\right)^{H-\frac{1}{2}}-\left(-t^{\prime}\right)^{H-\frac{1}{2}}\right]^{2} d t^{\prime}\right. \\
& \left.+\frac{1}{2 H}\right\}
\end{aligned}
$$

where $\Gamma$ is the Gamma function. The parameter $H \in(0,1)$ is called the Hurst exponent and the process is called a fractional Brownian motion (or $\mathrm{fBm}$ ). Ordinary Brownian motion is recovered as the special case where $H=\frac{1}{2}$.

The implication of Eq. (21) is that such a process does not (at least for $H \neq \frac{1}{2}$ ) consist of a sequence of independent Gaussian increments, but that the coefficient $\mathcal{V}_{H}$ is consistent with Lagrangian "memory" over previous increments.

For a given tracer trajectory, one can plot the growth of its variance as a function of time. A log-log plot of this variable, if it is associated with a $\mathrm{fBm}$ process, will determine 


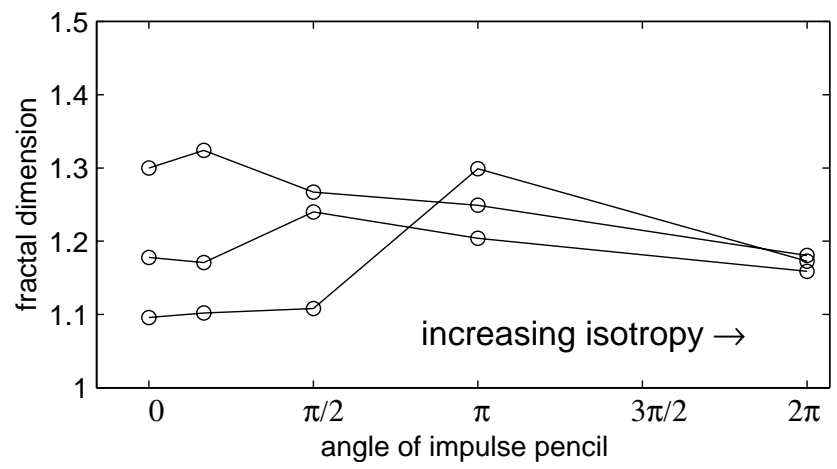

Fig. 10. Influence of alignment of impulse dipoles on the calculated fractal dimension.

the Hurst exponent. For a trajectory with isotropic displacments, this exponent bears a direct relationship to the fractal dimension of the path: this is

$D=\min \left[\frac{1}{H}, 2\right]$

(derived by Mandelbrot (1985)). For ordinary Brownian motion, the path has a fractal dimension of 2 (i.e. $H=\frac{1}{2}$ in Eq. 22). For motion that is strictly non-fractal (e.g. particles advected in a potential flow field), one expects a simple linear curve with $D=1$ (i.e. $H \uparrow 1$ in Eq. 22).

Thus, the limiting fractal dimension of $D_{L} \simeq 1.5$ associated with Fig. 4 could imply a fractional Brownian motion with Hurst exponent $H \simeq 2 / 3$. The variance of such a process (Eq. 20) should exhibit a $t^{4 / 3}$ time-dependence. The parameter $H$ can be determined directly from a numerically determined trajectory by applying regression to the logarithm of standard deviation on the logarithm of time, although a long time record is typically needed to achieve a converged estimate of this power (Rapaport (1985) discusses this in the context of molecular diffusion). We should also note that Eq. (20) represents a relationship that is true in ensemble average. Nevertheless, by way of demonstration, we plot the standard deviation of the trajectory shown in Fig. 4c as a function of time, and determine from this the Hurst exponent. This is carried out in Fig. 11, where we obtain a value of $H \simeq 0.64 \pm .04$ after 1000 time-steps.

\section{Conclusions}

We have proposed a simple physical mechanism to explain the generation of velocity fluctuations in the surface region of an ocean. This is based on the viscous exchange of impulse between the ocean and an evolving atmospheric boundary layer. The true geophysical phenomenon will be complicated by many additional dynamical considerations, such as temperature gradients, buoyancy, surface wave phenomena, coriolis effects, and ocean drift current. In the present model, we attempt to isolate a single physical component from this complex system, namely the aspect of tangential impulse exchange.
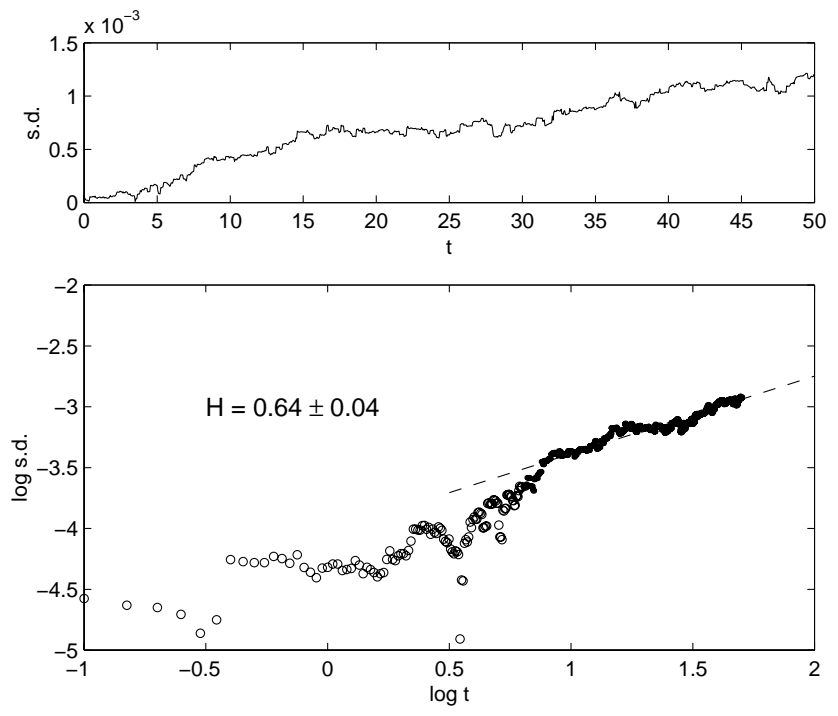

Fig. 11. Hurst exponent calculated from a plot of standard deviation against time (assuming fractional Brownian motion).

The ocean-atmosphere exchange of impulse is demonstrated through a numerical model in vertical section. The flow is represented numerically through the kinematics of impulse doublet sheets. From this we develop a threedimensional Lagrangian model of the advection and diffusion of impulse dipoles into a half-space ocean. In this threedimensional case, we represent the "atmosphere" by a continual generation of dipoles at the ocean surface. Our incentive for doing this is to model the fractal dimension of the trajectories of passive tracers in a lateral plane at a fixed ocean depth. The fractal dimension of such trajectories can be approximated numerically using the algorithm of Rapaport (1985) or alternatively, by understanding the process as a fractional Brownian motion.

For a scaled-down numerical example, we apply the model in order to understand the effect on the fractal dimension of truncating a record of a trajectory after a varying number of steps has elapsed. In addition, we examine the effect of resampling the trajectory location data at coarser intervals of time. We scale the problem up to the dimensions of a typical ocean trajectory, although we note that this proves to be computationally expensive.

In the literature, approaches to wind-induced ocean turbulence are usually based on the mechanism of wave propagation (see, for example, Sect. 4.3 of Kraus, 1972), and are derived from a treatment of momentum transfer at the ocean-air interface. The essential usefulness of the present approach, based on the exchange of impulse, follows from the relationship between impulse and vorticity (vorticity is the curl of impulse density). Thus, in order to impart impulse to the ocean, vorticity is generated. The resulting flow evolution is expressed through a nonlinear transport of a tangential impulse from the ocean surface. Such a process is complementary to that of wave formation (which is associated with the exchange and transport of normally-oriented impulse). The 
two processes may be superposed, and under this circumstance, they will almost certainly be coupled.

Thus, we introduce a numerical model in which wind flowing tangentially at the ocean surface becomes the causal agent for the creation of vortex doublet sheets. The ocean is represented as a fluid which, in the mean, is stationary; in this fluid, a fluctuation field (induced by these doublet elements) evolves into depth. When this evolving impulse distribution interacts with a passive tracer at depth, the tracer experiences translation in its fixed plane and, in this process, describes a fractal trajectory. We emphasize that the ocean itself does not undergo macroscopic drift in the present model.

We note that our impulse-based explanation for the fractal dimension of passive tracer trajectory poses an alternative to that derived from the considerations of dynamical systems theory. In various phenomenological theories of mesoscale turbulence, the ocean surface at such scale is considered to consist of ensembles of horizontal large-scale vortex eddies, i.e. with dipole moments oriented normally to the ocean surface; see, for example, DiBattista et al. (1998) or Provenzale (1999). Coherent vortical structures (which are typically three-dimensional) can originate from purely geostrophic mechanisms. In some circumstances, a lowdimensional system, consisting of such eddies, can exhibit deterministic chaos, and this can be characterized by a fractal dimension. If the cyclic variations of the system are rapid enough, one could conceive of eddy configurations which give rise to passive tracer paths of non-integer fractal dimension. However, such a mechanism seems highly contingent. A tracer in the presence of a slowly-rotating, large-scale eddy (or eddy-pair) could plausibly be expected to exhibit the integer dimension $(D=1)$ normally associated with an analytic curve arising from a harmonic (in this case, Biot-Savart) potential. Eddies generated by coriolis forces or by topographic stress form a visible part of the macroscopic flow at large scale. The question is whether such objects themselves constitute the near-surface fluctuation field, and whether they are responsible for the fractal dimension of tracers.

We are attracted to the alternative notion that a much larger stochastic system better represents the condition of the natural ocean. (The underlying contrast between the two approaches is analyzed in Osborne and Provenzale, 1989). We are drawn to the postulate that the near-surface fluctuation field is derived from a mechanism that is common throughout a significant spectrum of spatial scales, from $1 \mathrm{~m}$ to $10^{4} \mathrm{~m}$. This range would hint at the universality of structure generally observed in turbulence phenomena. This is to suggest that fractional Brownian motion in the ocean bears a scale relationship to eddy diffusivity in general, for example, in the meter-scale experiment reported by Richardson and Stommel (1948) and in the observations of fractional Brownian motion in smaller bodies of water first reported by Hurst (1951).

We note a number of alternative stochastic approaches to the question of passive tracers in turbulent flow. For example, a model equation can be postulated with a random forcing term (e.g. Carmona et al., 1997). In addition, one can postulate a specific scaling law and develop from this a Lagrangian model of turbulent transport (e.g. Viecelli , 1989). By way of contrast, the approach described in the present note does not invoke an explicit turbulence model, rather we seek to form a Lagrangian model of the equation of motion itself Eq. (4) and model its associated boundary conditions kinematically.

We draw attention to a further distinguishing feature of the present approach. We have described a specific viscous mechanism whereby vortex doublet sheets are continually generated at the ocean's surface. The implication of Eq. (4) is that these elements subsequently diffuse into the ocean: the initial direction of this diffusive flux is predominantly downward. As this diffusion proceeds, a tracer float submerged at a constant depth is constrained to inscribe its trajectory in a horizontal plane that is normal to this direction of flux. Although there is a continual movement of doublet elements passing normally through this trajectory plane, the sheet density on the plane remains, on average, stationary. Thus, as time advances, there is little significant mean time-evolution of sheet density in the drifter's plane of motion. This is in contrast with the case of a "free" tracer which is materially transported (passively and without constraint) within the same three-dimensional diffusing field. For similar reasons, the present case is distinguished from that of a tracer passively transported within a dispersing patch of point vortices in a two-dimensional plane.

The notion of a "fluctuation field" in the ocean with a kilometer spatial scale may seem to violate human intuition concerning the scale of a "fluctuation". The question is really one of perspective in the context of a continuum doublet sheet whose partitioning reveals the property of selfsimilarity (incidentally, this is in contrast to the case of a singlet vortex sheet). In effect, a small fragment of a large doublet sheet retains in miniature its integrity as a doublet sheet. The converse is also true: aggregates of uniform contiguous small-scale doublet elements, perceived at sufficiently large scale, are mathematically indistinguishable from a consolidated sheet.

At the "hundred kilometer scale", the notion that a fluctuation field in the ocean is "wind-driven" may also need elaboration. In this context, we understand "wind" to be the large-scale movement of the atmosphere relative to the ocean. What is being modelled at this scale is the viscous interaction of these two fluids at their mutual interface.

The fact that the estimates of fractal dimension that result from the present model broadly resemble those determined from geophysical experiment lends plausibility to the proposed viscous mechanism of tangential impulse-exchange at the ocean surface. As we have explained, the mechanism is robust (i.e. fractal dimension does not depend on specific configurations of macroscopic eddies); furthermore, it is spatially pervasive over the ocean surface, and it fits naturally above a hierarchy of similar phenomena occurring at progressively smaller scales.

Acknowledgements. This paper was originally presented at the 25 th General Assembly of the European Geophysical Society, Nice, France, 25-29 April 2000. 


\section{References}

Addison, P. S., Qu, B., Nisbet, A., and Pender, G.: A non-Fickian particle-tracking diffusion model based on fractional Brownian motion, Int. J. Numer. Meth. Fluids, 25, 1373-1384, 1997.

Batchelor, G. K.: An introduction to fluid dynamics, Cambridge Univ. Press, UK, 1967.

Beale, J. T. and Majda, A.: High order accurate vortex methods with explicit velocity kernels, J. Comput. Phys., 58, 188-208, 1985.

Bernard, P. S.: A deterministic vortex sheet method for boundary layer flow, J. Comput. Phys., 117, 132-145, 1995.

Buttke, T. F.: Lagrangian numerical methods which preserve the Hamiltonian structure of incompressible fluid flow, in: NASA ASI Series 395 (Ed) Beale, J. T., et al.) 301-306, 1993.

Buttke, T. F. and Chorin, A. J.: Turbulence calculations in magnetization variables, Appl. Num. Meth., 112, 47-54, 1993.

Carmona, R. A., Grishin, S., Xu, L., and Molchanov, S.: Surface stretching for Ornstein-Uhlenbeck velocity fields, Elect. Comm. in Probab., 2, 1-11, 1997.

Chorin, A. J.: Vorticity and turbulence, Springer Verlag, New York, 1994.

DiBattista, M. T., Majda, A. J., and Turkington, B.: Prototype geophysical vortex structures via large-scale statistical theory, Geophys. Astrophys. Fluid Dyn., 89, 235-283, 1998.

Fishelov, D.: A new vortex scheme for viscous flows, J. Comput. Phys., 86, 211-224, 1990.

Gustafson, K. and Sethian, J.: Vortex methods and vortex motion, SIAM, 1991.

Hurst, H. E.: Long-term storage capacity of resevoirs, Trans. Am. Soc. Civil Engrs., 116, 770-775, 1951.

Krasny, R.: Desingularization of periodic vortex sheet roll-up, J. Comp., 65, 292-313, 1986.

Kraus, E. B.: Atmosphere-ocean interaction, Oxford Univ. Press, UK, 1972.

Kuz'min, G. A.: Ideal incompressible hydrodynamics in terms of the vortex momentum density, Phys. Lett., 96A, 88-90, 1983.

Lamb, H.: Hydrodynamics, Cambridge Univ. Press, UK, 1932.

Lighthill, J.: An informal introduction to theoretical fluid mechanics, Oxford Univ. Press, UK, 1986.

Mandelbrot, B. B. and Van Ness, J. W.: Fractional Brownian motions, fractional noises and applications, SIAM Rev., 10, 422437, 1968

Mandelbrot, B. B.: The fractal geometry of nature, W. H. Freeman, San Francisco, 1977.

Mandelbrot, B. B.: Self-affine fractals and the fractal dimension, Physica Scripta, 32, 257-260, 1985.

Mandelbrot, B.B.: Multifractals and 1/f noise, Springer Verlag,
1999.

Osborne, A. R., Kirwan, A. D., Provenzale, A., and Bergamasco, L.: Fractal drifter trajectories in the Kuroshio extension, Tellus, 41A, 416-435, 1989.

Osborne, A. R. and Provenzale, A.: Finite correlation dimension for stochastic systems with power-law spectra, Physica D, 35, 357-381, 1989.

Osborne, A. R. and Caponio, R.: Fractal trajectories and anomalous diffusion for chaotic particle motions in 2D turbulence, Phys. Rev. Lett., 64, 1733-1736, 1990.

Oseledets, V. I.: On a new way of writing the Navier-Stokes equation: the Hamiltonian formalism, (Transl. Russ. Math. Surveys, 44, 210-211, 1989), Comm. Moscow Math. Soc., 1988.

Provenzale, A.: Transport by coherent barotropic vortices, Annu. Rev. Fluid Mech., 31, 55-93, 1999.

Rapaport, D. C.: The fractal nature of molecular trajectories in fluids, J. Stat. Phys., 40, 751-758, 1985.

Richardson, L. F.: The problem of contiguity, (also in Collected papers of Lewis Fry Richardson, vol. 2, 379-627, Cambridge Univ. Press, 1993), Gen. Syst. Yearbook, 6, 140-187, 1961.

Richardson, L. F. and Stommel, H.: Note on eddy diffusion in the sea, J. Met., 5, 238-240, 1948.

Roberts, P. H.: A Hamiltonian theory for weakly interacting vortices, Mathematika, 19, 169-179, 1972.

Russo, G. and Smereka, P.: Impulse formulation of the Euler equations: general properties and numerical methods, J. Fluid Mech., 391, 189-209, 1999.

Sanderson, B. G. and Booth, D. A.: The fractal dimension of drifter trajectories and estimates of horizontal eddy-diffusivity, Tellus, 43A, 334-349, 1991.

Smereka, P.: A Vlasov description of the Euler equation, Nonlinearity, 9, 1361-1386, 1996.

Summers, D. M.: A representation of bounded viscous flow based on Hodge decomposition of wall-impulse, J. Comput. Phys., 158, 28-50, 2000a.

Summers, D. M.: On the formation of vortices at a solid boundary, Proc. Roy. Soc. Lond., A 456, 1183-1192, 2000 b.

Summers, D. M.: Turbulent transport in the ocean based on impulse-exchange, Geophys. Res. Abst., 2, 2000c.

Summers, D. M.: Towards a Lagrangian model of boundary layer turbulence, Physica D, 154, 287-312, 2001.

Viecelli, J. A.: Structure of Lagrangian turbulence, Phys. Fluids A, 1, 1836-1843, 1989.

Voss, R.F.: Fractals in nature: from characterization to simulation, Ch. 2 in: The science of fractal images, (Ed) Barnsley, M. F., et al.), Springer-Verlag, 1988. 